\title{
TEMA 17-2015: Ganglio centinela en el melanoma cutáneo
}

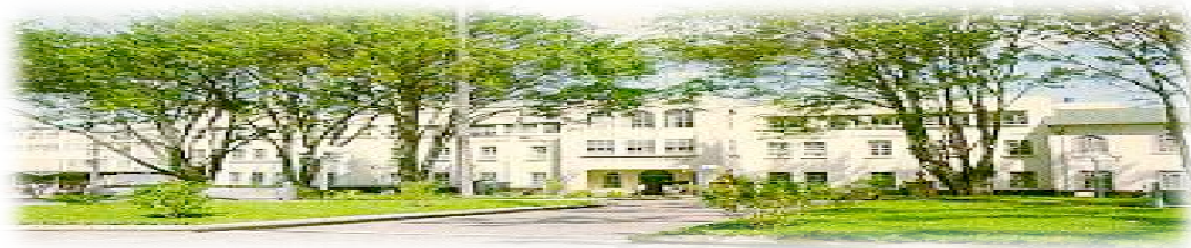

Hospital San Juan de Dios, San José, Costa Rica. Fundado en 1845

Recibido:

$12 / 07 / 2015$

Aceptado:

$24 / 08 / 2015$

Dra. Loretta Piccolo Johanning ${ }^{1}$

Dr. Eduardo Brenes Leñero ${ }^{2}$

${ }^{1}$ Médico Residente de Dermatología, Universidad de Costa Rica. Hospital San Juan de Dios. lpiccolo@medicos.cr

${ }^{2}$ Médico Residente de Cirugía General, Universidad de Costa Rica. Hospital México.

edbrenes@medicos.cr

\section{RESUMEN}

La biopsia del ganglio centinela se considera una herramienta diagnóstica con gran poder pronóstico y de estadiaje ya que permite detectar enfermedad nodal clínicamente negativa debajo del umbral de cualquier otra técnica no patológica (incluyendo modalidades de imágenes) $)^{(1,2,3,4,5,6)}$. Es el único examen diagnóstico con tanto alta sensibilidad como especificidad ${ }^{(1,6,7)}$.

Bajo este concepto se han desarrollado múltiples estudios comparando su utilidad como predictor de metástasis y mortalidad; tomando esto en consideración, varias guías han surgido.

\section{PALABRAS CLAVE}

Melanoma, ganglio centinela, biopsia.

\begin{abstract}
Sentinel node biopsy is considered a diagnostic tool with prognostic and staging power as it allows to detect clinically negative nodal disease below the threshold of any other nonpathological study (including imaging modalities $)^{(1,2,3,4,5,6)}$. It is the only diagnostic test with both high sensibility and specificity ${ }^{(1,6,7)}$.

Under this concept multiple studies that compare its usefulness in predicting metastasis and mortality have been developed; taking this into consideration, a series of guidelines have been created.
\end{abstract}

\section{KEY WORDS}

Melanoma, sentinel lymph node, biopsy. 


\section{DISCUSIÓN}

De la transformación maligna de los melanocitos, células pigmentadas especializadas

derivadas de la cresta neural localizadas a nivel de la unión dermoepidérmica, surge el melano$\mathrm{ma}^{(3,7,8,9,10,11)}$. Aunque solo corresponde a 5\%$10 \%$ de los cáncer de piel, es causante de hasta $75 \%$ de sus muertes, representando una importante carga económica y de salud ${ }^{(3,7,12)}$.

Una vez considerado como poco común, su incidencia y mortalidad han aumentado dramáticamente en las últimas décadas $(1,2,6,10,11,13,14,15,16,17,18,19,20,21,22)$. Según la Sociedad Americana de Cáncer la incidencia aumenta 5\%$7 \%$ por año en Estados Unidos $(3,4)$, con incidencias mayores en otros países como Australia, Nueva Zelanda, Noruega e Israel ${ }^{(10,19)}$. A medida que la incidencia aumenta, nuevas técnicas se han desarrollado para estadificar y tratar a estos pacientes $^{(23)}$.

Biopsia del Ganglio Centinela

La base ideológica de la biopsia del ganglio centinela (SLNB por sus siglas en inglés) recae en el concepto de que diferentes regiones de la piel tienen patrones específicos, usualmente ordenados y predecibles, de drenaje linfático a ganglios regionales, y que por una región determinada de la piel hay por lo menos un ganglio linfático específico (el ganglio o ganglios "centinela") en la cadena linfática al cual los vasos linfáticos aferentes drenan primero ${ }^{(3,11,16)}$.

A pesar de que diseminación hematógena tumoral puede ocurrir, la mayoría de los pacientes con melanoma presentarán metástasis ganglionares primero $^{(3,13)}$. De esta manera, el estado histológico del ganglio centinela refleja el estado de los ganglios remanentes de la misma cade$\mathrm{na}^{(3,4,5,7,11,16,22,24,25,26,27)}$.

La incidencia de "skip" metástasis (en el cual el ganglio centinela está libre de tumor pero los ganglios no centinela se encuentran con metástasis a la linfadenectomía) parece ser de $<5 \%{ }^{(5)}$.

\section{Técnica SLNB}

Todo paciente que presente una lesión sospechosa por melanoma debe de someterse a una biopsia excisional con márgenes estrechos preferiblemente negativos ${ }^{(1,2,6,28)}$. Debe evitarse un mayor margen para, de ser necesario, permitir realizar un SLNB subsecuente ${ }^{(1,2,6,28)}$. Idealmente el SLNB debe de realizarse al momento de la disección local amplia del melanoma primario ya que el ganglio centinela puede no ser preciso si es realizado luego de la escisión amplia en áreas donde hay un drenaje linfático ambiguo, o posterior a un colgajo local dado que la inyección de la solución puede ocurrir lejos del sitio primario verdadero $^{(1,6,7)}$.

Inicialmente para definir e identificar el ganglio centinela se buscaba la visualización del color azul en el ganglio luego de la inyección de un colorante en el sitio tumoral primario. Este colorante fue inicialmente isosulfán azul o Azul V Patentado, sin embargo por su baja disponibilidad algunos adoptaron el uso del azul de metileno para el mapeo linfático, a pesar de datos conflictivos respecto a su comparabilidad y seguridad. Se adoptó también una modalidad de enfoque dual, utilizando el radiocoloide marcado para detectar la localización del SLN vía linfocintigrafía e intraoperatoriamente utilizando una sonda portátil que detecta radiación gamma en conjunto con la visualización azul del ganglio $^{(3,7,24,25,29)}$. Actualmente no existe un radiocoloide ideal, por lo que el mismo varía a nivel mundial $^{(30)}$.

En Estados Unidos se ha mejorado la localización preoperatoria con el uso del SPECT-CT, permitiendo una mayor detección de compromiso metastásico, sin importar el sitio anatómi$\mathrm{co}^{(3,12)}$.

El ${ }^{99 \mathrm{~m}} \mathrm{Tc}$-Tilmanocept es un radiofármaco cuyo blanco es el receptor CD206 designado para la identificación del ganglio centinela. Permite identificar $98.7 \%$ de los ganglios, más que aquellos detectables por el colorante azul ${ }^{(30)}$.

Una vez identificado este ganglio o ganglios centinela, se remueven y evalúan por enfermedad metastásica seccionándolos, y utilizando técnicas estándares de hematoxilina y eosina(H\&E) combinadas con tinciones de inmunohistoquímica tales como S100, HMB-45 y/o Melan-A si la $\mathrm{H} \& \mathrm{E}$ es negativa o inconclusa. Esto aumenta la sensibilidad y ocasiona un mayor estadiaje en $10-20 \%$ de los pacientes ${ }^{(1,6,13)}$. Dado que los macrófagos, pigmentados o no, pueden semejarse al melanoma metastásico en el ganglio centinela, la inmunohistoquímica es de gran utili$\operatorname{dad}^{(25)}$.

No se recomienda la biopsia por congelación ya que destruye la posibilidad de analizar el nódulo con inmunohistoquímica, es menos sensible que la examinación postoperatoria del tejido fijado con formalina, y puede afectar las pocas células malignas presentes. Cuando se realiza, presenta una sensibilidad del 34\% y una tasa de falsos negativos del $12 \%^{(8,3,31)}$.

\section{Objetivos SLNB}

El uso del SLNB para un estadiaje regional pre- 
ciso tiene varios beneficios: 1) proporciona información importante al médico tratante $\mathrm{y}$ al paciente a la hora de evaluar opciones terapéuticas subsecuentes; 2) permite identificar pacientes con metástasis ganglionares que podrían verse beneficiados de linfadenectomía temprana; 3) identifica pacientes que pueden ser candidatos para tratamientos adyuvantes como IFN $\alpha 2 \beta$ y radioterapia; 4) permite la identificación de pacientes aptos a inscribirse en ensayos clínicos; y 5) determina pronóstico ${ }^{(26,6,32)}$.

Aproximadamente $80 \%$ de los pacientes con melanoma cutáneo primario van a tener el ganglio centinela negativo por tumor ${ }^{(17,22)}$. El objetivo de esta técnica es detectar el 15\%-20\% de los pacientes con melanoma cutáneo primario $\mathrm{y}$ ganglios clínicamente negativos que sí tendrán melanoma metastásico en dicho ganglio..$^{(13,16,25,33)}$.

\section{$\underline{\text { Avances }}$}

El manejo de los ganglios regionales en el melanoma ha sido controversial desde que Snow, en 1892 propusiera realizar una linfadenectomía total electiva para todos los pacientes con melanoma sin importar si existiese evidencia clínica de metástasis regionales nodales. De esta manera al remover los ganglios linfáticos de manera temprana en el proceso de metástasis, la enfermedad no progresaría a sitios distantes. Desde entonces se han realizado una serie de estudios que demuestran que mientras unos pacientes no se beneficiarían de una linfadenectomía total de rutina al no tener metástasis regionales nodales, hay un grupo de pacientes que sí lo harían ${ }^{(14,24)}$. En 1992, Donald L. Morton publicó el primer reporte de la técnica de biopsiar el ganglio centinela. Bajo el concepto de que la mayoría de los pacientes estadio I sometidos a linfadenectomía de rutina no tenían metástasis ganglionar, desarrolló un nuevo procedimiento que permitía la identificación intraoperatoria del ganglio centinela utilizando colorantes ${ }^{(34)}$.

En 1994, Morton et al iniciaron un estudio controlado randomizado multicéntrico para establecer la precisión del estadio ganglionar basados en el SLN, y así evaluar el impacto del manejo inicial con SLNB en pacientes que presentaban un melanoma localizado. Esto con el fin de determinar si el vaciamiento ganglionar inmediato en los pacientes con ganglios centinela positivos por tumor podría mejorar la sobrevida. En este estudio, denominado "Primer Ensayo Multicéntrico de Linfadenectomía Selectiva" (MSLT-I por sus siglas en inglés), los pacientes se dividieron en dos grupos: aquellos que fueron sometidos a una escisión amplia más ganglio centinela, con linfadenectomía inmediata si el SNB era positivo (60\% los pacientes, considerados el grupo biopsia), y aquellos que fueron sometidos a escisión amplia más observación, con linfadenectomía retardada en caso de evidencia clínica de recurrencia nodal durante el seguimiento (40\% de los pacientes, considerados el grupo observacional). Dentro de los pacientes randomizados a la biopsia, 16\% tuvieron SNB positivo por tumor. En los siguientes 10 años, un 4\% adicional desarrolló recurrencia nodal. Dentro del grupo de pacientes randomizados a la observación, la incidencia cumulativa de recurrencia nodal alcanzó un $16 \%$ a los 5 años y un $20 \%$ a los 10 años, sugiriendo así que las metástasis nodales progresan eventualmente a un tamaño palpable de no ser removidas ${ }^{(24,28,35,36)}$.

Evidenciaron también que los pacientes dentro del grupo biopsia que presentaban metástasis en ganglio centinela presentaron peores resultados que aquellos pacientes con ganglios libres de tumor. El estatus del ganglio centinela fue entonces el mayor predictor de recurrencia o muerte por melanoma.

El MSLT-I validó el uso de la biopsia por ganglio centinela en pacientes con melanomas de grosor intermedio y mayor, aumentando el control de la enfermedad regional $\mathrm{y}$, en aquellos pacientes que presentan metástasis nodales, parece mejorar sustancialmente la sobrevida por melanoma $^{(24)}$.

La importancia del SLNB en pacientes con melanomas delgados (de $1.2 \mathrm{mmm}$ o menos) no fue valorada específicamente en este estudio ya que el número de pacientes con dicho grosor fue muy pequeño como para poder realizar conclusiones referentes al efecto terapéutico del tratamiento basado en biopsia ${ }^{(28,37)}$.

El MSLT-I demostró también que $88 \%$ de los pacientes con un solo ganglio afectado por tumor no presentan metástasis nodales adicionales cuando se examinaban los ganglios de la linfadenectomía subsiguiente a la H\&E. Esto sugiere que si las metástasis nodales se limitan a una o dos ganglios centinelas, la SLNB podría ser tanto diagnóstica como terapéutica. Con esta idea en mente, se desarrolló la segunda fase de estos estudios, el MSLT-II, con la hipótesis de que se podría evitar la linfadenectomía en la mayoría de los pacientes con metástasis a ganglios centinela ${ }^{(35,38)}$.

El MSLT-II se encuentra en progreso ${ }^{(37)}$. Es un estudio randomizado donde se está comparando la utilidad de la SLNB con vaciamiento ganglionar (CLND) versus SLNB con observación de los ganglios linfáticos por medio de ultrasonido 
(que permite detectar metástasis de incluso 4 $\mathrm{mm}$ ). El principal resultado esperado es determinar la sobrevida relacionada al melanoma. Resultados secundarios incluyen sobrevida en total y libre de enfermedad, precisión diagnóstica de la histopatología y de los marcadores moleculares e inmunológicos, y calidad de vida ${ }^{(35)}$. Los participantes del MSLT-II se seguirán durante un periodo de 10 años. Está estimado a finalizar en setiembre del $2022^{(37)}$.

Con el objetivo de analizar cuáles factores se asociaban con enfermedad ganglionar adicional, Lee et $\mathrm{al}^{(18)}$ realizaron un estudio con 1599 participantes para determinar cuáles pacientes podrían verse beneficiados del CLND. De estos, $24 \%$ presentaron ganglios no centinela positivos $\mathrm{y}$ observaron que el grosor del tumor primario (Breslow $\geq 3 \mathrm{~mm}$ ), el sitio primario (principalmente tronco y extremidades), el tamaño tumoral del ganglio centinela $(\geq 2 \mathrm{~mm})$ y el número de ganglios centinela positivos por tumor estuvieron significativamente asociados con positividad tumoral de ganglios no centinela, siendo el grosor primario y el mayor tamaño tumoral del ganglio centinela los que correlacionaron mejor. No encontraron que la presencia de ulceración, el índice mitótico, el tipo histológico ni el género fueran determinantes.

Scoggins et $\mathrm{al}^{(39)}$ asociaron la localización del tumor en una extremidad, el nivel Clark y la invasión linfovascular con un mayor riesgo de SLN positivo por tumor. La positividad por tumor en el ganglio centinela junto con el género masculino, mayor grosor Breslow, y ulceración lo asociaron con una menor sobrevida.

McMasters et al ${ }^{(40)}$ estudiaron 2000 pacientes con melanoma cutáneo de por lo menos $1 \mathrm{~mm}$ de grosor evaluando ganglios centinela en tránsito (fuera de las bases linfáticas tradicionales ) en busca de metástasis. Identificaron 62 pacientes $(3.1 \%)$ con ganglios en tránsito; de estos, 13 (21\%) contenían metástasis y en el caso de 11 pacientes $(84.6 \%)$ era el único sitio con metástasis ganglionares. Concluyeron que, a pesar de que los ganglios centinela en tránsito se identifican infrecuentemente, contienen enfermedad metastásica casi tan frecuentemente como los ganglios centinela en las bases ganglionares cervicales, axilares e inguinales. Esto demuestra la importancia de la linfocintigrafía prequirúrgica y la búsqueda intraoperatoria meticulosa por ganglios centinela en tránsito.

La posibilidad de presentar un ganglio centinela positivo por tumor en pacientes con melanoma cutáneo primario $\leq 1 \mathrm{~mm}$ es de aproximadamente $5 \%$, por lo que el uso del SLNB es de controver- sia ya que aunque el porcentaje de pacientes que presentarían metástasis nodales en este grupo es muy bajo, sí hay un cierto grupo de pacientes que podrían beneficiarse de la técnica ${ }^{(4,13)}$. Aquellos con tumor $0.76-1.00 \mathrm{~mm}$, presentan un riesgo es de 6-11\%; los que presentan un tumor $<0.76 \mathrm{~mm}$, la posibilidad es de $2-4 \%$ o menos, sin importar si son T1a o T1b; en grosores $1-2 \mathrm{~mm}$ es de $15-20 \%$; en grosor de $2-4 \mathrm{~mm}$ es de $25-35 \%$; y en mayores de $4 \mathrm{~mm}$ es de un $40-50 \%{ }^{(1,4,28)}$. Por esto, algunos consideran al nivel del grosor Breslow como la variable pronóstica más importante en predecir la sobrevida en pacientes con melanoma cutáneo, ${ }^{(20)}$ y como el mejor predictor de riesgo metastásico ${ }^{(29,41)}$.

Han et $\mathrm{al}^{(42)}$ intentaron determinar cuáles factores clinicopatológicos pudiesen ser predictivos de metástasis a ganglio centinela en el melanoma delgado $(\leq 1 \mathrm{~mm})$. La única recomendación que pudieron hacer es que el ganglio centinela se debe realizar en pacientes con melanomas $\geq$ $0.75 \mathrm{~mm}$, pero no para los de menor grosor. Encontraron que factores como la presencia de ulceración o un nivel Clark de IV predecían enfermedad en ganglio centinela en tumores $\geq 0.75 \mathrm{~mm}$, y no consideraron a la tasa mitótica como una variable pronostica importante, aún en pacientes con por lo menos $0.75 \mathrm{~mm}$ de grosor $^{(23,42)}$.

Ulmer et $\mathrm{al}^{(43)}$ a su vez analizaron 1834 ganglios de 1027 pacientes con melanoma con ganglios negativos al ultrasonido que fueron sometidos a biopsia del ganglio centinela entre el 2000 y el 2008 utilizando distintas técnicas de histopatología como lainmunohistoquímica e inmunocitología cuantitativa. Para la inmunocitología registraron el número de células cancerígenas (positivas por el antígeno gp100) diseminadas por millón de células del ganglio linfático (densidad "DCC" por sus siglas en inglés), y demostraron que a mayor densidad DCC, mayor riesgo de muerte por melanoma. Pacientes con densidad DCC positiva $\leq 3$ tuvieron un mayor riesgo de muerte por melanoma en comparación con pacientes con densidad DCC $=0$. Presentaron un aumento del riesgo de muerte del melanoma en $6 \%$ a 5 años. Determinaron a la densidad DCC como un factor predictor de muerte más fuerte que la histopatología. Consideraron entonces que la densidad DCC, junto con el grosor del tumor primario y la presencia de ulceración presentan un impacto sinergístico en la sobrevida del paciente, y los consideraron como los factores pronóstico más importantes.

Berk et $\mathrm{al}^{(15)}$ confirmaron la asertividad y el valor pronóstico del SLNB en el melanoma primario, y 
su uso como herramienta válida de estadiaje en melanomas $\geq 1 \mathrm{~mm}$ al igual que la baja tasa de recurrencia nodal regional en pacientes con SLNB negativo. Consideraron el estado del ganglio centinela como el factor predictor más importante de recurrencia, y la ulceración del tumor primario como el mayor factor predictor de positividad del ganglio centinela y como predictor independiente de recurrencia tumoral.

Nagaraja et $\mathrm{al}^{(44)}$ buscaron identificar las variables clínicopatológicas más predictivas de metástasis a ganglios no centinela cuando el ganglio centinela está positivo por melanoma. Encontraron que la presencia de ulceración, satelitosis, neurotropismo, invasión angiolinfática, localización extensa, macrometástasis $>2 \mathrm{~mm}$, extensión extranodal, compromiso capsular y la presencia de más de un ganglio centinela positivo fueron factores asociados con una alta probabilidad de metástasis a ganglios no centinela.

Ante tanta controversial, los estudios MSLT-II y MINITUB que se encuentran en progreso, pueden ser de ayuda para determinar cuales pacientes podrían evitar la linfadenectomía de manera segura $^{(44)}$.

El estudio MINITUB (Minimal SN Tumor Burden) es un estudio prospectivo observacional que compara CLND versus observación en un grupo seleccionado de pacientes de melanoma primario T2-T3 de grosor intermedio $(1.01-4 \mathrm{~mm})$ con ganglio centinela positivo, pero con carga tumoral limitada dentro del ganglio centinela. En el cáncer de mama, se ha demostrado que pacientes con mínima carga tumoral en el ganglio centinela pueden ser manejados de manera segura solo con observación nodal, sin ser sometidos a CLND. Se cree que en melanoma esto podría también ser cierto, pero debe ser validado por lo que este estudio se encuentra realizando este registro. Se realizará seguimiento de los pacientes por 10 años, por lo que está estimado a terminar para el $2023^{(13,45,46)}$.

\section{Guías}

Las guías del 2011 de la Academia Americana de Dermatología recomiendan realizar el SNLB en lesiones que presenten un grosor mayor a $1 \mathrm{~mm}$. Aquellos con grosores menores a $1 \mathrm{~mm}$ presentan mayor controversia ${ }^{(2)}$.

Las guías recientes de la NCCN (National Comprehensive Cancer Network) del $2014^{(28)}$ no recomiendan realizar SLNB para pacientes con melanoma in situ (estadio 0). Tampoco recomiendan realizarlo para estadios IA o IB que son muy delgados $(0.75 \mathrm{~mm}$ o menos) a menos que se encuentre otro factor de alto riesgo; en ese caso queda a decisión del paciente y el médico tratante.

Sí recomiendan realizarlo en pacientes con estadio IA que presentan melanomas de 0.76 $1.0 \mathrm{~mm}$ de grosor, sin embargo esto no es un consenso dado que el grado de positividad en estos pacientes es poco claro. Para pacientes en estadio IB o II, el SLNB debe de ser discutido con el paciente.

El SLNB podría ser considerado además para pacientes con enfermedad en-tránsito solitaria y resecable en estadio III, sin embargo su impacto en la sobrevida del paciente es poco clara.

En pacientes que de otra forma serían candidatos a SLNB, la decisión de no realizarlo debe basarse en comorbilidades o preferencia del paciente. En caso de no estar disponible la técnica de la SLNB, las guías actuales de la NCCN no recomiendan realizar una disección ganglionar electiva de rutina en pacientes que presenten ganglios clínicamente negativos pero que se consideren en riesgo de metástasis regionales. En estos casos se podría considerar una resección amplia del tumor o la referencia a un centro clínico donde la técnica de la SLNB sí se encuentre disponible.

Según recomendaciones de la NCCN, si el ganglio centinela está negativo, no está indicado realizar una disección ganglionar regional. Aquellos que sí presenten la SLNB positiva, deben ofrecerse la alternativa de realizar la disección ganglionar. No hay suficientes estudios que respalden la observación de la base ganglionar como opción, por lo que no se recomienda ${ }^{(28)}$.

\section{Otras recomendaciones}

Ha surgido la duda sobre el potencial aumento de riesgo de desarrollo de metástasis en tránsito a la hora de realizar la biopsia de ganglio centinela o de la linfadenectomía a causa de la obstrucción del flujo por disrupción mecánica de los linfáticos. Sin embargo, varios estudios han demostrado que el desarrollo del melanoma en tránsito depende de la biología del tumor (grosor tumoral, ulceración en el tumor primario, tumor primario en pierna, o metástasis del ganglio centinela), y no del enfoque quirúrgico que se le de a los ganglios linfáticos regionales ${ }^{(3)}$.

Ciertas recomendaciones se basan en el tipo de melanoma, no solo en los factores de riesgo presentes. Respecto al melanoma lentigo maligno, se recomienda realizar el ganglio centinela en lesiones mayores a $1 \mathrm{~mm}$ de grosor, al igual que en melanoma de extensión superficial y melanoma nodular ${ }^{(11)}$. El melanoma tipo nodular fue encontrado como un factor predictor de positivi- 
dad del ganglio centinela por Cadili et $\mathrm{al}^{(41)}$. Por otro lado, en el caso del melanoma acrallentiginoso, se requiere del ganglio centinela en la mayoría de los $\operatorname{casos}^{(9,11)}$.

Sin embargo, se considera que el riesgo de metástasis nodales es en general independiente del subtipo clínico, excepto en el caso del melanoma desmoplásico. Se ha observado que pacientes con melanoma desmoplásico mixto presentan mayor riesgo de metástasis que aquellos con melanoma desmoplásico puro, por lo que algunos sugieren que se podría diferir el uso de la SLNB en estos pacientes, siendo este enfoque todavía controversial ${ }^{(13,28,47)}$.

Cuando se trata de melanomas en cabeza y cue1lo, debido a que los patrones de drenaje linfático en dicho sitio son múltiples y variables, el número y la localización de los ganglios centinela resulta difícil de predecir, por lo tanto realizar una biopsia excisional de ganglio linfático puede ser desafiante ${ }^{(5,32,40,48,49)}$. Por esto, ha surgido cierta duda sobre la confiabilidad del uso de la SLNB en esta localización. Se han reportado tasas de recurrencia de $0-25 \%$ en melanomas de cabeza y cuello aún con SLNB negativo ${ }^{(32)}$. $\mathrm{Pa}-$ reciera que las lesiones en cuero cabelludo $\mathrm{y}$ cuello posterior se comportan más agresivamente que lesiones de la cara, y pareciera que los pacientes con melanoma primario de cabeza y cuello tienen peores resultados que aquellos con melanomas primarios en otros sitios del cuer$\mathrm{po}^{(50)}$. En estos pacientes el uso de SPECT/CT (tomografia con emisión de positrones y tomografía computarizada) se asocia con mejores tasas de identificación del ganglio centinela ${ }^{(12,13)}$, el cual se logra identificar en $99.7 \%$ de los casos de los pacientes que se someten a SLNB ${ }^{(13)}$. En este caso la linfoscintigrafía prequirúrgica es una herramienta importante para guiar la resección completa de todos los ganglios centinela ${ }^{(5,49)}$.

En el caso de melanomas de región periorbitaria, no se ha demostrado relación entre el estado del ganglio centinela y la invasión perineural del tumor $^{(51)}$.

Bajo ciertas circunstancias, el uso del SLNB no se justifica. Es innecesario realizarlo en aquellos pacientes que se presenten con enfermedad sistémica. Similarmente cuando se presentan con un ganglio clínicamente palpable; este debería de ser sometido a aspiración con aguja fina inicialmente. Cuando se han sometido a una escisión local amplia previamente con reconstrucción es controversial dado que surge la duda sobre si los linfáticos han sido alterados. No se han hecho recomendaciones sobre su uso cuando ha habido un SLNB previo, pero no pareciera ser una contraindicación absoluta ${ }^{(31)}$.

\section{Complicaciones SLNB y linfadenectomía}

Las principales ventajas de la SLNB sobre la linfadenectomía total son el menor número y menor grado de efectos adversos, y la selección de un menor número de ganglios linfáticos que pueden ser analizados extensamente por el patólogo vía H\&E e inmunohistoquímica ${ }^{(25,52,53)}$. Complicaciones de la herida (infección, dehiscencia de la herida, dolor, parestesias, y formación de seromas o hematomas) y complicaciones regionales (linfedema, tromboflebitis, dolor, parestesias, y pérdida de rangos de movimiento) se podrían presentar, sin embargo son más frecuentes en pacientes que se someten a la linfadenectomía ${ }^{(3,8)}$. Reacción alérgica al tinte azul se ha reportado, pero su incidencia es de un $0.2 \%{ }^{(3)}$.

Pareciera ser que las complicaciones más frecuentes posterior a SLNB son la formación de seromas $(1.2 \%)$, lesiones nerviosas transitorias $(0.9 \%)$ e infecciones menores de la herida quirúrgica $(0.9 \%)$. En contraste, linfedemacrónico $(7.8 \%)$ e infecciones de la herida $(6.5 \%)$ son las complicaciones más comunes en pacientes que fueron sometidos a CLND.

El número de ganglios resecados y la colocación de un drenaje de succión cerrado aumentan el riesgo de complicaciones en pacientes sometidos a SLNB pero no a $\mathrm{CLND}^{(52)}$.

\section{CONCLUSIONES}

La biopsia del ganglio centinela ha reemplazado la disección linfática ganglionar electiva para la evaluación de metástasis linfáticas nodales regionales en pacientes con melanoma cutáneo primario $^{(52)}$ y se ha convertido en un procedimiento de gran importancia para el estadiaje y tratamiento para estos pacientes. Considerado como mínimamente invasivo, es de gran exactitud a la hora de identificar a pacientes con metástasis nodales ${ }^{(39)}$.

Se ha determinado entonces al estado de los ganglios linfáticos regionales como el factor pronóstico más importante en la sobrevida y recurrencia del melanoma, ${ }^{(1,2,6,7,15,16,33,40,44,54)}$ siendo un factor determinante de terapia $y$ pronóstico $^{(1,2,7,21,41)}$. 


\section{BIBLIOGRAFÍA}

1. Bailey E Sober A Tsao H Mihm M Johnson T. Cutaneous Melanoma: en Fitzpatrick's Dermatology in General Medicine. Editorial Mc Graw Hill Companies, Inc. Estados Unidos de América. 8va edición, 2012;Pp: 14251427.

2. Bichakjian CK. Halpern AC Johnson $\mathrm{TM}$ et al. Guidelines of care for the management of primary cutaneous melanoma. J Am AcadDermatol 2011;65:1032-47.

3. Gershenwald JE Ross MI. SentinelLymph-Node Biopsy for Cutaneous Melanoma. N Eng J Med 2011;364:173845.

4. Gershewald JE Coit DG Sondak VK Thompson JF. The Challenge of Defining Guidelines for Sentinel Lymph Node Biopsy in Patients with Thin Primary Cutaneous Melanoma. Ann SurgOncol 2012:1-3.

5. Phan GQ Messina JL Sondal VK Zager JS. Sentinel Lymph Node Biopsy for Melanoma: Indications and Rationale. Cancer Control 2009;16(3):234-239.

6. Bailer E Sober AJ Tsao H Mihm MC Johnson TM. Chapter 124: Cutaneous Melanoma.En Fitzpatrick'sDermatology in General Medicine. 8va Edición. http://accessmedicine.mhmedical.com.e zproxy.sibdi.ucr.ac.cr:2048/content.asp $\underline{x}$ ?bookid $=392 \&$ sectionid $=41138842 \& j$ umpsectio-

$\underline{\mathrm{nID}=41153922 \& \text { Resultclick }=2}$ Accesad o 25 mayo, 2014.

7. Ludgate MW Johnson TM Wang TS. Chapter 100: Skin Cancer. En Hazzard's Geriatric Medicine and Gerontology, 6ta Edición. http://accessmedicine.mhmedical.com.e zproxy.sibdi.ucr.ac.cr:2048/content.asp $\underline{x}$ ?bookid $=371 \&$ sectionid $=41587719 \& j$ umpsectio-

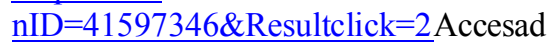
o 20 mayo, 2014.

8. Heistein Jonathan B Melanoma. Medscape. Abril, 2014. http://emedicine.medscape.com/article/ 1295718-overview.Accesado 14 mayo, 2014.
9. Piccolo L Chan A. Melanoma AcralLentiginoso: una entidad a tener en cuenta en la práctica diaria. $\mathrm{Rev} \mathrm{Cl}$ EMed UCR. Vol 3, No VI, 2013. Pp:1519.

10. Tan Wiston W. Malignant Melanoma. Medscape. Abril, 2014 http:/emedicine.medscape.com/article/ 280245-overview . Accesado 14 mayo, 2014.

11. Section 12. Melanoma Precursors and Primary Cutaneous Melanoma. In: Wolff K, Johnson R, Saavedra AP. eds. Fitzpatrick's Color Atlas and Synopsis of Clinical Dermatology, 7 ma edición. http://accessmedicine.mhmedical.com.e zproxy.sibdi.ucr.ac.cr:2048/content.asp $\mathrm{x}$ ?bookid $=682 \&$ Sectionid $=45130143$. Accesado 14 Mayo, 2014.

12. Stoffels I Boy C Pöppel, T et al. Association Between Sentinel Lymph Node Excision With or Without Preoperative SPECT/CT and Metastatic Node Detection and Disease-Free Survival in Melanoma. JAMA. 2012;308(10):10071014.

13. Durham AB Wong SL. Sentinel Lymph Node Biopsy in Melanoma. Controversies and Current Guidelines. Future Oncol. 2014, 10(3):429-42.

14. Lee JH Essner, $\mathrm{R}$ Torisu-Itakura $\mathrm{H} \mathrm{Wa}$ nek L Wang H Morton DL. Lymph Nodes After Tumor-Positive Sentinel Lymph Node Dissection for Melanoma. J ClinOncol 2001;22:3677-3684.

15. Berk, DR Johnson DL Uzieblo A Kierman, M Swetter SM. Sentinel Lymph Node Biopsy for Cutaneous Melanoma. TheSanfordExperience, $\quad 1997$ 2004.ArchDermatol. 2005;141:10161022.

16. Heiken, TJ. The Role of Sentinel Node Biopsy in Skin Cancer. Medscape. Marzo, 2014. http://emedicine.medscape.com/article/ 1126050-overview.Accesado 20 Mayo, 2014.

17. Elias N Tanabe KK .Sober AJ et al. Is Completion Lymphadenectomy After a Positive Sentinel Lymph Node Biopsy for Cutaneous Melanoma Always Necessary? ArchSurg. 2004;139:400-405. 
18. Lee JH Essner R Torisu-Itakura H Wanek, L Wang H Morton DL. Factors Predictive of Tumor-Positive Nonsentinel Lymph Nodes After Tumor-Positive Sentinel Lymph Node Dissection for Melanoma. J ClinOncol 2004;22:36773684.

19. Kim KB Davies MA Rapini RP Hwu, P Bedikian AY. Chapter 39: Malignant Melanoma. En The MD Anderson Manual of Medical Oncology, 2da edición. http://accessmedicine.mhmedical.com.e zproxy.sibdi.ucr.ac.cr:2048/content.asp x?bookid $=379 \&$ sectionid $=39902069 \& j$ umpsectio$\underline{\mathrm{nID}}=39908241 \&$ Resultclick $=2$ Accesad o: 16 mayo, 2014.

20. Cole P Heller L Bullocks J Hollier LH. Stal, S. Chapter 16: The Skin and Subcutaneous Tissue. En Schwartz's Principles of Surgery, 9na edición. http://accessmedicine.mhmedical.com.e zproxy.sibdi.ucr.ac.cr:2048/content.asp $\underline{x}$ ?bookid $=352 \&$ sectionid $=40039758 \& j$ umpsectionID $=40043331 \&$ Resultclick $=2$ Accesad o 25 mayo, 2014.

21. Polk HC Qadan. M. Chapter 30: Malignant Melanoma and Squamous Cell Carcinoma of the Skin. En: Fischer's Mastery of Surgery. 6ta edición. Editorial Wolters Kluwer Health. Pp: 394400.

22. Sabel MS. Chapter 44: Oncology. En: CURRENT Diagnosis \& Treatment: Surgery. 13va edición. http://accesssurgery.mhmedical.com.ez proxy.sibdi.ucr.ac.cr:2048/content.aspx ?boo-

kid $=343 \&$ sectionid $=39702832$ \&jumpse ctionID $=39716887 \&$ Resultclick $=2$. Accesado: 26 mayo, 2014.

23. Goydos JS. Who Should Be Offered a Sentinel Node Biopsy for Melanoma Less Than $1 \mathrm{~mm}$ in Thickness? J ClinOncol 2013;31(35):4385-4386.

24. Morton DL Thompson JF Cochran AJ et al. Final Trial Report of SentinelNode Biopsy versus Nodal Observation in Melanoma.N Engl J Med 2014;370:599-609.

25. Prieto VG Curry JL. Sentinel Lymph Node Biopsy in Melanoma Pathology.
Medscape. Octubre, 2013. http://emedicine.medscape.com/articlel 1977004-overview. Accesado 21 Mayo, 2014.

26. Stone M. Evaluation and treatment of regional lymph nodes in melanoma. UpToDate. $\quad$ Febrero 2014. http://www.uptodate.com.ezproxy.sibdi. ucr.ac.cr:2048/contents/evaluation-andtreatment-of-regional-lymph-nodes-inmelano-

ma? source $=$ search result\&search $=$ evalu ation + and + tratment + of + regional + lymp $\mathrm{h}+$ nodes $+\mathrm{in}+$ melanoma\&selectedTitle $=$ 1\%7E150.Accesado 22 Mayo, 2014.

27. Martí E Díaz AJ Martín M et al. El ganglio centinela en el melanoma: nuestra experiencia. CirPlasIberolatinoam. 2006;32(2):117-126.

28. NCCN Clinical Practice Guidelines in Oncology. Melanoma. Versión 4.2014 http://www.nccn.org/professionals/phys ician gls/PDF/melanoma.pdf. Accesado 15 Mayo, 2014.

29. Urba WJ Washington CV Nadiminti H. Chapter 87: Cancer of the Skin. En Harrison'sPrinciples of Internal Medicine, 18va edición. http://accessmedicine.mhmedical.com.e zproxy.sibdi.ucr.ac.cr:2048/content.asp $\mathrm{x}$ ?bookid $=331 \&$ sectionid $=40726823 \mathrm{Ac}$ cesado 25 mayo, 2014.

30. Sondak VK. King DW Zager JS et al. Combined Analysis of Phase III Trials Evaluating ${ }^{99 m} \mathrm{Tc} / T$ Tilmanocept and $\mathrm{Vi}$ tal Blue Dye for Identification of Sentinel Lymph Nodes in Clinically NodeNegative Cutaneous Melanoma. Ann SurgOncol 2013;20:680-688.

31. Keidan RD Fong P Kestenberg WL. Sentinel Lymph Node Biopsy in Patients With Melanoma. Medscape. Abril, 2014. http://emedicine.medscape.com/article/ 854424-overview\#showall. Accesado 20 Mayo, 2014.

32. Schmalbach CE Nussenbaum B Rees RS Schwartz J Johnson TM Bradford CR. Reliability of Sentinel Lymph Node Mapping With Biopsy for Head and Neck Cutaneous Melanoma. ArchOtolaryngol Head NeckSurg. 2003;129:61-65.

33. Valsecchi ME Silbermins D de Rosa N Wong SL Lyman GH. Lymphatic Map- 
ping and Sentinel Lymph Node Biopsy in Patients With Melanoma: A MetaAnalysis.J ClinOncol. 2011;29:14791487.

34. Morton DL Wen DR Wong JH et al. Technical details of intraoperative lymphatic mapping for early stage melanoma. Arch. Surg. 1992;127(4):392399.

35. Morton DL. Overview and Update of the Phase III Multicenter Selective Lymphadenectomy Trials (MSLT-I and MSLT-II) in Melanoma.ClinExp Metastasis 2012;29(7):699-706.

36. Morton DL Thompson JF Cochran AJ et al. Sentinel-Node Biopsy or Nodal Observation in Melanoma. $\mathrm{N}$ Eng $\mathrm{J}$ Med 2006;355:1307-17.

37. U.S. National Institutes of Health. Clinical Trials. Multicenter Selective Lymphadenectomy Trial II (MSLT-II). Abril, 2014.

http://clinicaltrials.gov/show/NCT0029 7895. Accesado 17 Mayo, 2014.

38. Nelson R. Lack of Clear Evidence for Sentinel Node Bioppsy in Melanoma? Medscape. $\quad 2013$. http://www.medscape.com/viewarticle/7 77518.Accesado 21 Mayo, 2014.

39. Scoggins CR Bowen AL Martin RC et al. Prognostic Information From Sentinel Lymph Node Biopsy in Patients With Thick Melanoma. ArchSurg 2010;145(7):622-627.

40. McMasters KM Chao C Wong SL et al. IntervalSentinelLymphNodes in Melanoma. ArchSurg. 2002;137:543-549.

41. Cadili, A.; Dabbs, K. Predictors of sentinel lymph node metastasis in melanoma. J can chir. 2010;53(1):32-36.

42. Han D Zager JS Shyr, Y et al. Clinicopathological Predictors of Sentinel Lymph Node Metastasis in Thin Melanoma. J ClinOncol 2013;31:4387-4393.

43. Ulmer A Dietz K Hodak I et al. Quantitative Measurement of Melanoma Spread in Sentinel Lymph Nodes and Survival. PLoSMed 2014;11(2):e1001604, doi:10.1371/journal.pmed.1001604.

44. Nagaraja V Eslick GD. Is complete lymph node dissection after a positive sentinel lymph node biosy for cutaneous melanoma always necessary? A metaanalysis. EJSO 2013;39:669-680.

45. European Organization for Research and Treatment of Cancer. Melanoma Group. EORTC 1208: MINITUB Registration Study.Octubre, 2013. http://www.melanomagroup.eu/site/data basefor-melanoma-group-

member/minitub.html. Accesado 18 Mayo, 2014

46. U.S. National Institutes of Health. Clinical Trials. Minimal SN Tumor Burden (Minitub). Setiembre, 2013. http://clinicaltrials.gov/show/NCT0194 2603.Accesado 20 Mayo, 2014.

47. Ariyan CE Coit DG. Clinical aspects of sentinel lymph node biopsy in melano$m a$.SeminDiagnPathol 2008;25(2):8694.

48. Carlson GW Murray DR Greenlee R et al. Management of Malignant Melanoma of the Head and Neck Using Dynamic Lymphoscintigraphy and Gamma Probe-Guided Sentinel Lymph Node Biopsy. ArchOtolaryngol Head NeckSurg. 2000;126:433-437.

49. Fincher TR O'Brien JC McCarty TM et al. Patterns of Drainage and Recurrence Following Sentinel Lymph Node Biopsy for Cutaneous Melanoma of the Head and Neck.ArchOtolaryngol Head NeckSurg. 2004;130:844-848.

50. Agnese DM Maupin R Tillman B Pozderac RD Magro C Walker MJ. Head and Neck Melanoma in the Sentinel Lymph Node Era. ArchOtolaryngol Head NeckSurg. 2007;133(11):1121 1124.

51. Esmaeli B. Sentinel Lymph Node Biopsy Is Not Relevant to Perineural Invasion for Eyelid Melanomas. ArchOphthalmol 2008;26(11):1607.

52. Roaten JB Pearlman N Gonzalez R Gonzalez R McCarter MD. Identifying Risk Factors for Complications Following Sentinel Lymph Node Biopsy for Melanoma.ArchSurg 2005;140:85-89.

53. Wrone DA Tanabe KK Cosimi B Gadd MA Souba WW Sober AJ. LymphedemaAfterSentinelLymphNodeBiopsyforCutaneous Melanoma. A report of 5 cases. ArchDermatol. 2000;136:511514. 
54. Dubois RW Swetter SM Atkins M et al. Developing Indications for the Use of Sentinel Lymph Node Biopsy and Adjuvant High-Dose Interferon Alfa- $2 b$ in Melanoma. ArchDermatol. 2001;137:1217-1224.

\section{CONFLICTO DE INTERÉS Y/O AGRADE-} CIMIENTOS

Los autores declaran que no existió ningún conflicto de interés en el presente reporte. 